\title{
Belief change and argumentation in multi-agent scenarios
}

\author{
Jürgen Dix ${ }^{1}$ (D) . Sven Ove Hansson ${ }^{2}$. \\ Gabriele Kern-Isberner $^{3}$. Guillermo R. Simari ${ }^{4}$
}

Published online: 17 October 2016

(C) Springer International Publishing Switzerland 2016

\section{Introduction}

The present special issue is based on work presented at the Belief Change and Argumentation in Multi-Agent Scenarios Seminar, held on June 3rd - 7th, 2013, at Schloss Dagstuhl, Wadern, Germany. ${ }^{1}$ The papers in this issue profited from the discussions and interactions during this productive event: they are the result of a Call for papers that we put together after the seminar. From the ten originally submitted papers, after a careful reviewing process (each paper was evaluated by at lerast three reviewers), five were finally accepted and found their way into this special issue.

The Dagstuhl seminar brought together researchers from (1) Multiagent Systems, (2) Belief Revision, and (3) Argumentation: these three important fields of research contribute different perspectives on an array of problems that elicit important new research questions that can be addressed combining insights obtained in those areas.

Research on multiagent systems has produced increasingly relevant modeling results characterizing a general paradigm for distributed problem solving (particularly in resource bounded domains) and for the understanding of cooperation among autonomous intelligent agents. Usually, agents acting in uncertain and dynamic scenarios need to have the capability of reasoning defeasibly about the environment, 1.e., the agents should be capable of revising

${ }^{1}$ http://www.dagstuhl.de/en/program/calendar/semhp/?semnr=13231

Jürgen Dix

dix@tu-clausthal.de

1 Clausthal University of Technology, 38678 Clausthal-Zellerfeld, Germany

2 Royal Institute of Technology, 11428 Stockholm, Sweden

3 Technische Universität Dortmund, 44227 Dortmund, Germany

4 Universidad Nacional del Sur in Bahía Blanca, Buenos Aires, 8000, Argentina 
hypotheses or previously made conclusions to maintain an adequate and consistent model of the world.

On the other hand, belief change theory formally considers all kind of epistemic changes in agent's knowledge in view of new information or the perception of changes in the world; this field of inquiry emerged from the basic AGM-theory of belief revision, thus named after Alchourrón, Gärdenfors, and Makinson who first structured the related ideas in 1985. The area has been substantially expanded to deal with different change operations for a variety of frameworks such as classical logics, conditional and default logics, logic programming and Horn clause logic, as well as probabilistic logic and ontologies, with close relationships to plausible reasoning.

Argumentation theory also introduces frameworks for defeasible reasoning, by setting up formal structures that allow the consideration and evaluation of arguments in favor of or against a certain option; here, the attention is focussed on the process of dialectical deliberation and on finding justifications for epistemic and practical decisions. For that reason, argumentation theory is particularly useful for decision making, both in a single agent reasoning and in a social environment that involves a group of agents. The area also offers natural methodological frameworks for distributed and collaborative decision making, or for solving conflicts between agents, by evaluating opposite statements and views. Proposed approaches to argumentation range from very abstract argumentation frameworks to specialized argumentation systems, for instance for applications in law.

Belief revision and argumentation can be looked upon as two dimensions of reasoning that provide complementary possibilities; meanwhile belief revision investigates methods and quality criteria that address the problem of how beliefs should be changed or adjusted in a rational way, argumentation seeks for justifications that show why beliefs are established and options should be chosen. By combining them in different ways and producing different reasoning architectures, researchers are able to address both dimensions of reasoning and solve problems that out of reach of the formalisms and techniques produced in either area of research. Furthermore, and more significanty, because reasoning and decision making are two core components in agent architectures, closer links between these two components will bring about improvements of the agent model. Undoubtedly, and from a theoretical point of view, considering the connections between argumentation and belief revision will cross fertilize both areas bringing about advances in the scientific field of knowledge representation.

The Dagstuhl seminar of which this special issue is a result, has brought together researchers from the fields of argumentation theory and belief change theory, both from philosophy and computer science, to report innovative research results stem from the exchange of ideas aimed at combining argumentation and belief change in multiagent environment. Studies of the relations between both areas have started in recent years, with the aim to advance the state of the art within one field with methods and techniques of the other.

In the paper Two axiomatic characterizations for the system of spheres-based (and the Epistemic Entrenchment-based) multiple contractions by Maurício D. L. Reis, Pavlos Peppas, and Eduardo Fermé, the authors present two novel axiomatic characterizations using intuitive postulates for those multiple contraction functions.

The work described in A series of revisions of David Poole's specificity by Claus-Peter Wirth and Frieder Stolzenburg, introduce some new specificity orderings over Poole's specificity relation are presented and an efficient workaround the problems in those orderings for applications is suggested. 
In Belief revision in structured probabilistic argumentation model and application to cyber security by Paulo Shakarian, Gerardo I. Simari, Geoffrey Moores, Damon Paulo, Simon Parsons, Marcelo A. Falappa, and Ashkan Aleali, propose a probabilistic structured argumentation framework which extends Presumptive Defeasible Logic Programming with probabilistic models, and for belief revision they introduce a set of rationality postulates characterizing the belief revision operators including representation theorems stating the equivalence between classes of operators and their associated postulates demonstrating how the framework can be used to address the attribution problem in cyber security/cyber warfare.

The focus of Assessing the epistemological relevance of Dung-style argumentation theories by Gregor Betz is put on the evaluation from an epistemological point of view of Dung's theory of abstract argumentation frameworks. The author concludes that this theory is epistemologically problematic since does not provide a normatively adequate model for the evaluation of rational, multi-proponent controversy.

Finally, in the paper How to deal with unbelievable assertions by Matti Nykänen, Raul Hakli, and Satu Eloranta, the authors study the problem that occurs when an agent receives unbelievable information and propose two possible solutions that modify the information making it believable; one of the solutions requires that the sender adjusts the information using an explanation from the receiver, and in the alternate solution which arises from the impossibility of cooperation, the receiver revises the information making it acceptable in the context of its own beliefs.

We would like to express our appreciation to the authors which submitted their work, to the reviewers who provided insightful and constructive comments, and also to all the attendees to the Dagstuhl seminar where the discussions took place.

We are convinced that the quality of the work done by all these researchers will greatly contribute to the expansion of research on the topics that were at the center of the seminar and motivated this special issue. 